\title{
Corrigendum
}

\section{Corrigendum to "Investigation and Validation of Detection of Storage Stability of Difenoconazole Residue in Mango"}

\author{
Fangfang Zhao $\mathbb{D}^{1,2,3,4}$ Jingkun Liu, ${ }^{1,2,3,4}$ Bingjun Han, ${ }^{1,2,3,4}$ and Jinhui Luo ${ }^{1,2,3,4}$ \\ ${ }^{1}$ Analysis \& Testing Center, Chinese Academy of Tropical Agricultural Sciences, Xueyuan Road 4, Haikou 571101, Hainan, China \\ ${ }^{2}$ Laboratory of Quality \& Safety Risk Assessment for Tropical Products (Haikou), Ministry of Agriculture, Xueyuan Road 4, \\ Haikou 571101, Hainan, China \\ ${ }^{3}$ Hainan Provincial Key Laboratory of Quality and Safety for Tropical Fruits and Vegetables, Xueyuan Road 4, Haikou 571101, \\ Hainan, China \\ ${ }^{4}$ Quality Supervision and Inspection Center of Tropical Agro-Products, Xueyuan Road 4, Haikou 571101, Hainan, China \\ Correspondence should be addressed to Fangfang Zhao; zhaofangfangfang@163.com
}

Received 19 May 2019; Accepted 23 May 2019; Published 16 June 2019

Copyright ( 2019 Fangfang Zhao et al. This is an open access article distributed under the Creative Commons Attribution License, which permits unrestricted use, distribution, and reproduction in any medium, provided the original work is properly cited.

In the article titled "Investigation and Validation of Detection of Storage Stability of Difenoconazole Residue in Mango" [1], the authors' contribution sentence in the Disclosure should be removed as Dr. Fangfang Zhao should be listed as the only first author.

\section{References}

[1] F. Zhao, J. Liu, B. Han, and J. Luo, "Investigation and validation of detection of storage stability of difenoconazole residue in mango," Journal of Food Quality, vol. 2019, Article ID 5641643, 8 pages, 2019. 


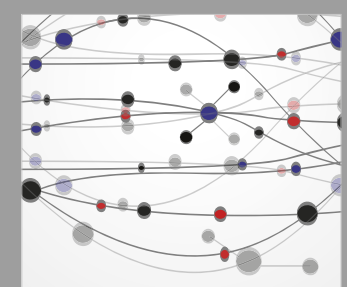

The Scientific World Journal
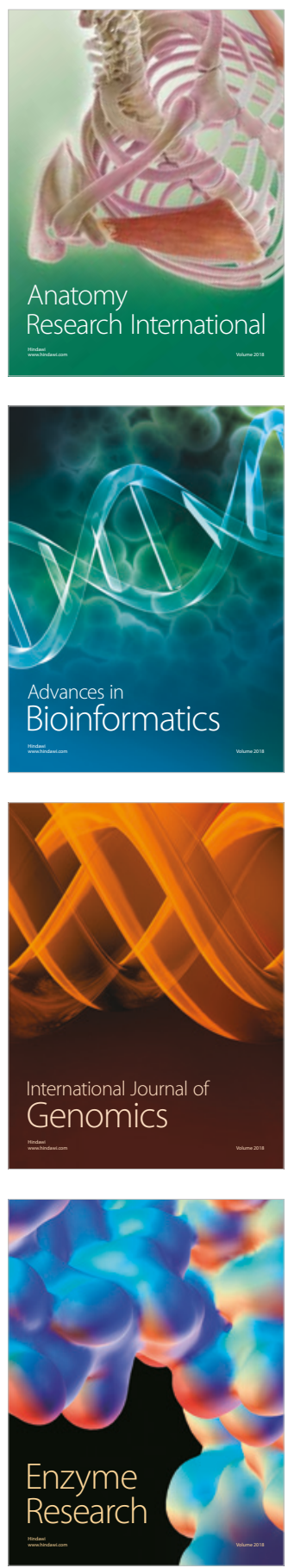
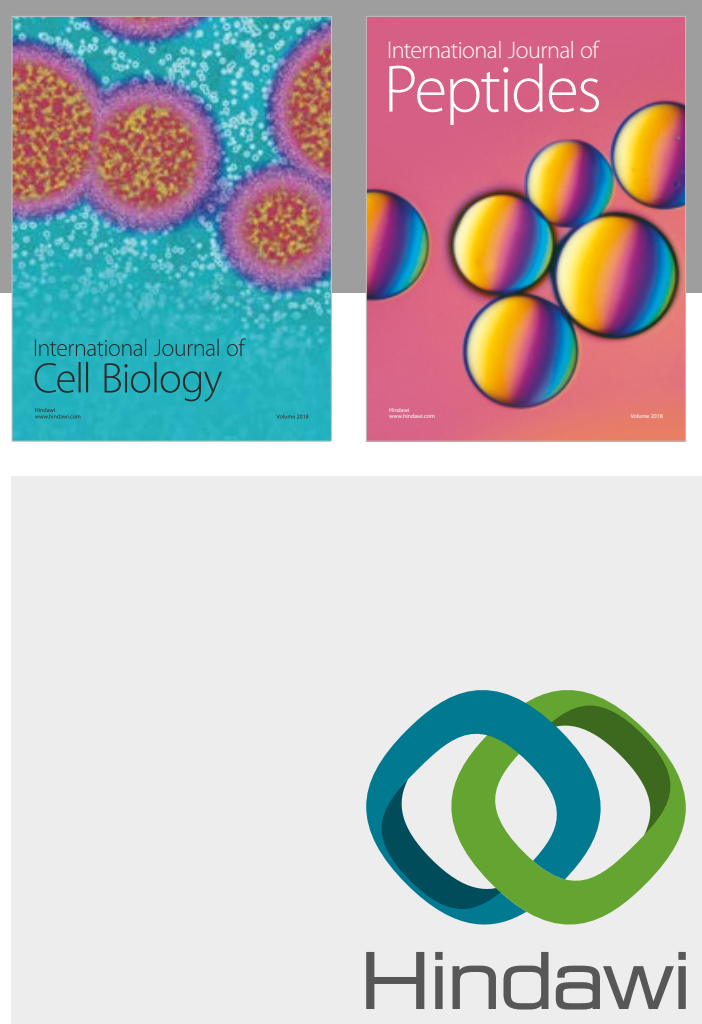

Submit your manuscripts at

www.hindawi.com
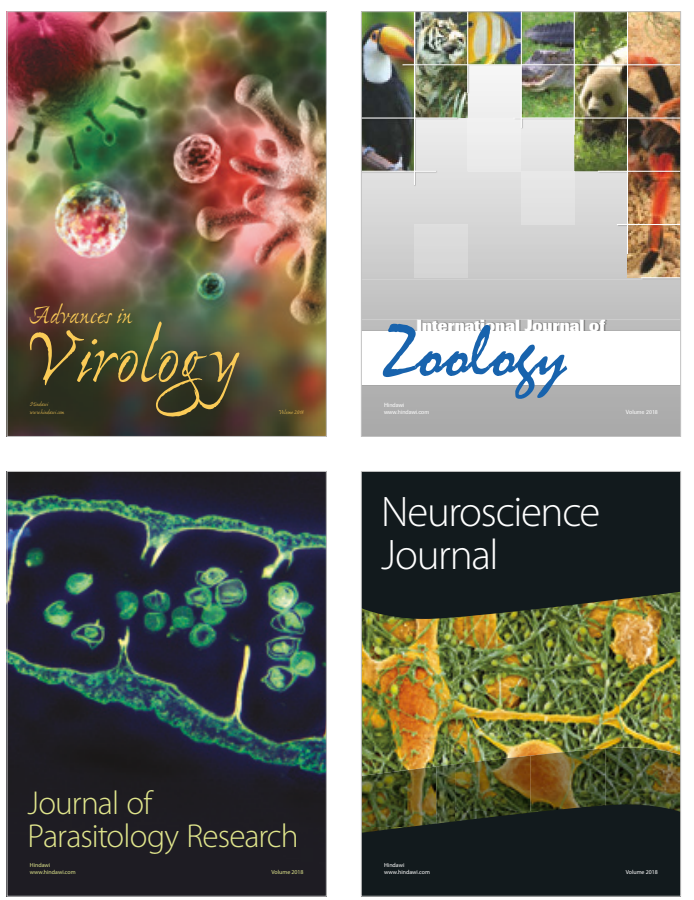
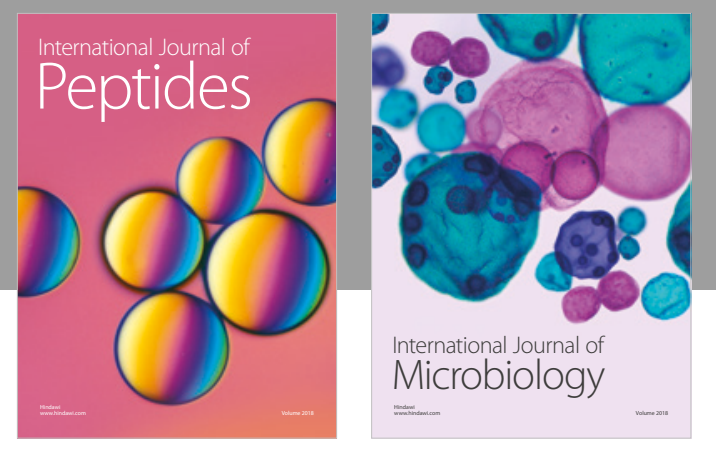

nternational Journal of Microbiology
Journal of

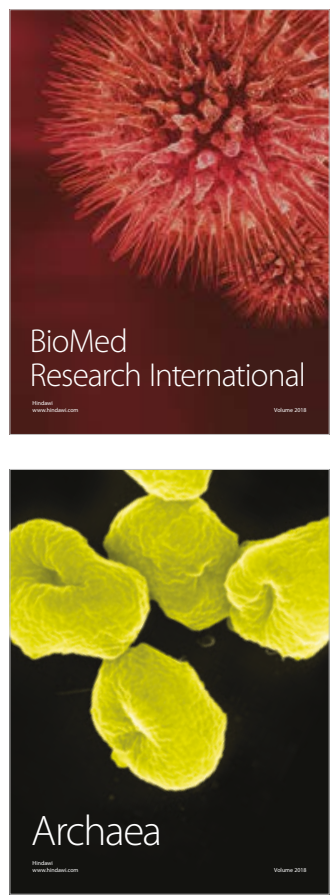